\title{
INVESTIGATION OF THE RELATIONSHIP OF TNFRSF11A GENE POLYMORPHISMS WITH BREAST CANCER DEVELOPMENT AND METASTASIS RISK IN PATIENTS WITH BRCA1 OR BRCA2 PATHOGENIC VARIANTS LIVING IN THE TRAKYA REGION OF TURKEY
}

\author{
Özdemir K ${ }^{1}$, Gürkan $\mathrm{H}^{1, *}$, Demir S ${ }^{1}$, Atli E ${ }^{1}$, Özen $\mathrm{Y}^{1}$, Sezer A², Tunçbilek N ${ }^{3}$, Çicin $\mathrm{I}^{4}$ \\ *Corresponding Author: Hakan Gürkan, MD, PhD, Department of Medical Genetics, Genetic Diseases \\ Diagnosis Center, Trakya University Faculty of Medicine, Balkan Campus, 22030 Edirne, Turkey. Tel: \\ +90-533-218-8005. Fax:+90-284-235-7641. Email: dr_hakangurkan@yahoo.de, hgurkan@trakya.edu.tr
}

\begin{abstract}
Modifying genes play an exclusive role in the genetic regulation of the risk of breast cancer development in women with a pathogenic variation of $B R C A 1$ or $B R C A 2$. Therefore, it has been suggested that TNFRSF11A, which is among those modifying genes present in breast cancer development, may have a significant role in patients with positive $B R C A 1$ or $B R C A 2$ variations. In our study, we investigated the probable effects of single nucleotide polymorphisms (SNPs) in the TNFRSF11A gene, such as rs4485469, rs9646629, rs34739845, rs17069904, rs 884205 , rs 4941129 on the risk of breast cancer in patients with $B R C A 1$ or $B R C A 2$ variations. A total of 23 breast cancer patients with pathogenic variations in the $B R C A 1$ or $B R C A 2$ genes, 28 patients with no pathogenic variations in the $B R C A 1$ or $B R C A 2$ genes, and 55 healthy women as a control group, were included in this study. The SNPs were determined with allelic discrimination analysis through the real-time polymerase chain reaction (qPCR) method. There was no statistically significant difference between the SNPs of the TNFRSF11A gene rs4485469, rs9646629, rs34739845, rs17069904, rs884205, rs4941129 and metastasis, estrogen receptor, progesterone receptor and CerB2 receptor positivity between patient and control group ( $p$ $>0.05$ ). However, the rs4485469 SNP was found to be

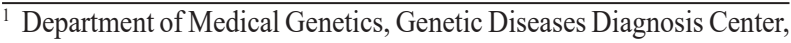
Trakya University Faculty of Medicine, Edirne, Turkey

${ }^{2}$ Department of General Surgery, Faculty of Medicine, Trakya University, Edirne, Turkey

${ }^{3}$ Department of Radiology, Faculty of Medicine, Trakya University, Edirne, Turkey

${ }^{4}$ Department of Medical Oncology, Faculty of Medicine, Trakya University, Edirne, Turkey
}

borderline significant between the patient groups with and without BRCA1 or BRCA2 mutations $(p=0.059)$. In patients with $B R C A 1$ or $B R C A 2$ pathogenic variations living in the Trakya region of Turkey, we could not determine the relationship between TNFRSF11 SNPs with breast cancer risk.

Keywords: $B R C A 1$; $B R C A 2$; Breast cancer; Single nucleotide polymorphism (SNP); TNFRSF11A gene.

\section{INTRODUCTION}

Although some progress has been made in understanding the role of the high-risk breast cancer susceptibility genes $B R C A 1$ and $B R C A 2$, what exactly has caused the variation of risk observed among mutation carriers remains unclear [1]. The risk of developing breast cancer varies widely among pathogenic variation carriers of $B R C A 1$ or $B R C A 2$. This apparent variability in cancer risk between $B R C A 1$ and $B R C A 2$ pathogenic variation carrier families and among individuals within families can be explained by modifying genes that partially affect mutation penetration [2]. In the microarray studies of irradiated lymphoblastoid cell lines from pathogenic variation carriers of $B R C A 1$ or $B R C A 2$, new genes have been found that affect and alter the expression of certain other genes. Such genes are called modifying genes. These modifying genes modulate penetrance, dominance, pleiotropy, or expression in individuals with Mendelian features [3]. Again, in some studies, it has been observed that genotype-phenotype correlations do not comply with intra-individual risk variations within mutation carrier families. Accordingly, if there is no significant correlation between mutant $B R C A$ alleles and phenotypes, it has been suggested that the risk of cancer can be modulated with other genetic and environmental factors [4]. Extensive international cohort studies show 
that these genes can increase the risk of breast cancer [2]. In the literature, many different regulatory loci have been proposed, including the nuclear receptor coactivator 3 (NCOA3, AIB1) involved in hormone metabolism, the androgen receptor $(A R)$ gene and the $R A D 51$ gene involved in DNA repair [5-7].

TNFRSF $11 A$ is a member of the tumor necrosis factor (TNF) receptor superfamily, which includes 32 members. The extracellular part of TNFRSF11A is a signal peptide that consists of 28 amino acids, with a total of 616 amino acids in the transmembrane protein and 21 amino acids in the short transmembrane and large cytoplasmic portions. The TNFRSF11A gene is localized in chromosome 18q21.33 and has 12 exons in total [8]. It has been determined that this receptor, which controls osteoclastogenesis and calcium metabolism, is expressed on the surface of macrophage/monocytic cells, $\mathrm{T}$ and $\mathrm{B}$ lymphocytes, fibroblasts, dendritic cells, chondrocytes, trophoblasts and precursor-mature osteoclasts [9]. The protein synthesis of TNFRSF11A has also been shown in some cancer cells, including those of breast and prostate cancer, with both being types of cancer with high potential for bone metastasis. TNFRSF11A is the only receptor that can maintain the binding TNF superfamily member 11 receptor activator of nuclear factor $\kappa \mathrm{B}$ ligand $(R A N K L)$ to preosteoclasts [10].

The RANKL gene encodes one of the members of the TNF ligand family that has 18 members and it is the key mediator of bone resorption. The encoded protein is a 317-amino acid peptide composed of two cellular and biologically active soluble forms that are membrane bound [11]. It has been demonstrated with messenger RNA (mRNA) studies that RANKL gene expression can occur in tissues of the lymph nodes, thymus, lung, spleen, brain, heart, intestine, kidney, liver, skeletal muscle, placenta, testicle, skin, breast, bone marrow, active T-lymphocytes and osteoblasts [12]. TNFRSF11A does not have the ability to activate protein kinases spontaneously like other TNF receptors. Therefore, after binding of RANKL, TNF receptor-related factors can bind to the cytoplasmic part of TNFRSF $11 A$ and activate intracellular signaling pathways. $R A N K L$ has crucial effects on the immune system as well as an osteoporotic effects [13]. Furthermore, preclinical studies in mice have shown that RANKL is also expressed in breast epithelial cells during pregnancy, and it is essential in mammary gland development, lactational hyperplasia of breast epithelial cells and milk production $[14,15]$. The expression of some malignant tumor cells in TNFRSF $11 \mathrm{~A}$ as well as $R A N K L$, has suggested that they may play a role in the stimulation of tumor cell proliferation [16].

TNFRSF 11A signals can become active in progenitor cells from core cells believed to be in $B R C A 1$ or $B R C A 2$ mutation carriers with breast cancer [17]. Intracellular signaling mediated by TNFRSF $11 \mathrm{~A}$ forms the basis of mammary gland development and regulates stem and progenitor cell divisions. Overexpression of TNFRSF11A promotes the abnormal proliferation of breast epithelial cells and prevents differentiation, which increases the incidence of tumorigenesis. As a result, dysfunctional mammary glands that have lobuloalveolar structures can be observed. In line with the underlying breast processes, the increased signals of TNFRSF11A promote breast cancer formation $[8,18]$.

In their study, Sigl et al. [17] have reported that TNFRSF 11A signaling can play an exclusive role in breast carcinogenesis guided by $B R C A 1$ or $B R C A 2$ mutations. In order to prove the accuracy of this hypothesis, they suggested two important conclusions by conducting a versatile analysis of the RANKL/TNFRSF11A system in a clinical setting. First, TNFRSF11A and RANKL are highly expressed only by breast cancer cells with $B R C A 1$ or BRCA2 mutations, and TNFRSF11A protein levels show a significant correlation with tumor grade in this scenario. Second, common TNFRSF11A polymorphisms that increase TNFRSF11A expression levels are associated with an increased risk of developing breast cancer in women with the BRCA1 or BRCA2 mutations. Therefore, it has been suggested that TNFRSF11A has a role in the aetiology of breast cancer caused by the BRCA1 or BRCA2 mutation [17]. In our study, we examined the effects of rs4485469, rs9646629, rs34739845, rs 17069904, rs884205 and rs4941129 single nucleotide polymorphisms (SNPs) in the TNFRSF11A gene in terms of the risk of breast cancer development.

\section{MATERIALS AND METHODS}

A total of 106 individuals were included in the study, of which 51 were patients diagnosed with breast cancer and 55 were healthy controls. The average age of those in the group diagnosed with breast cancer was 44.8 , while the average age of those in the healthy control group was determined to be 44.0 .

Of the 51 breast cancer patients who were included in the patient group of the study, a total of 23 female patients had been living in the Trakya region of Turkey for at least three generations, were not related to each other, were diagnosed with breast cancer and were determined to have $B R C A 1$ and/or $B R C A 2$ mutations; 28 female patients presented without a $B R C A 1$ and/or $B R C A 2$ mutation.

Of the 55 women in the control group, who volunteered to participate in the study, all were also living in the Trakya region for at least three generations, were not related to each other, were older than 18 years of age, were 
not diagnosed with familial breast cancer, had no history of breast-related disease during previous examinations and did not have any complaints about their breasts and ovaries. The Informed Volunteer Consent Form was signed by both the patients and the individuals in the control group, which contained information about the study and certified their approval. The study was approved by the Trakya University Faculty of Medicine Scientific Research Ethics Committee. The decision number was 16/03 with the numbered document TÜTF-BAEK 2016/219 on September 28, 2016 after the reason, purpose, approach and methods of the study were examined.

Peripheral venous blood samples of $2.5 \mu \mathrm{L}$ were drawn from the patients and those in the control groups, and genomic DNA was extracted using an EZ1 Advanced XL (Qiagen GmbH, Hilden, Germany) nucleic acid isolation device with the EZ1 DNA Blood $200 \mu \mathrm{L}$ isolation kits (Qiagen $\mathrm{GmbH}$ ). The concentration and purity values of the genomic DNA samples were measured with the Nano Drop 2000C device (Thermo Fisher Scientific, Wilmington, DE, USA) at wavelengths of 260/280 nm. Genomic DNA samples with an absorbance value at 1.52.0 and a concentration value of $20.0-80.0 \mathrm{ng} / \mu \mathrm{L}$ were included in the study. Then, allelic discrimination was performed through a real-time polymerase chain reaction (qPCR) process using the TaqMan ${ }^{\circledR}$ SNP Genotyping Assay kit (Applied Biosystems, Foster City, CA, USA) for rs4485469, rs9646629, rs34739845, rs17069904, rs884205 and rs4941129 SNPs.

Statistical Analyses. In the study, patient and control group data were analyzed with the Statistical Package for the Social Sciences (SPSS) version 16.0 software (SPSS Inc., Chicago, IL, USA). Demographic data of the patient and control groups were interpreted by obtaining the numbers $(n), \%, \chi^{2}$, mean, minimum and maximum values, and averages.

The genotype frequencies of the rs4485469, rs9646629, rs34739845, rs17069904, rs884205 and rs4941129 SNPs in the TNFRSF11A gene of the breast cancer patients (with and without $B R C A 1$ or $B R C A 2 \mathrm{mu}-$ tations) and the control group were analyzed. In addition, TNFRSF11A rs4485469, rs9646629, rs34739845, rs17069904, rs884205, rs4941129 SNPs genotypes were compared according to metastasis, estrogen receptor (ER), progesterone receptor (PR), CerbB2 receptor positivity status of the patients with and without BRCA1/BRCA2 mutation.

\section{RESULTS}

A total of 106 individuals, consisting of 51 patients (23 patients with $B R C A 1$ or BRCA2 mutations and 28 patients without $B R C A 1$ or $B R C A 2$ mutations) and 55 controls, were included in the study. The demographic information of the patients (with and without $B R C A 1$ or $B R C A 2$ mutations) and those in the control group is provided in Table 1.

There were no statistically significant differences in the genotype frequencies of the rs4485469, rs9646629, rs34739845, rs17069904, rs884205 and rs4941129 SNPs in the TNFRSF11A gene between the patients (with and without BRCA1 or BRCA2 mutations) and those in the control group $(p>0.05)$ (Table 2$)$. There were also no statistically significant differences detected in the genotype frequencies of the rs9646629, rs34739845, rs17069904, rs884205 and rs4941129 SNPs in the TNFRSF11A gene between the patient group with and without BRCA1 or $B R C A 2$ mutations $(p>0.05)$. However, rs4485469 SNP was found to be borderline significant between the patient groups with and without $B R C A 1$ or $B R C A 2$ mutations ( $p$ $=0.059)$ (Table 3 ).

Metastasis is a part of the clinical staging of patients with breast cancer, and the development and function of hormone receptors, estrogen and progesterone and CerbB2 oncoprotein that is one of the molecular prognostic factors, are important in terms of determining the prognosis and treatment method for the disease. These prognostic factors were used to identify the clinical and biological behavioral differences in patients with breast cancer and those in the high-risk group for whom the disease can develop rapidly. In our study, the results of metastasis, estrogen, progesterone and cerbB2 assessment, which were requested for routine examination in the patient group, were also evaluated.

Table 1. The demographic data of the patients (with and without BRCA1 or BRCA2 mutations) and the control group.

\begin{tabular}{|l|c|c|c|c|}
\hline Parameters & $\boldsymbol{n}$ & Mean \pm SD & Minimum & Maximum \\
\hline Patient group with $B R C A 1$ or $B R C A 2$ mutations & 23 & $45.13 \pm 13.73$ & 24.00 & 76.00 \\
\hline Patient group without $B R C A 1$ or $B R C A 2$ mutations & 28 & $44.54 \pm 8.28$ & 33.00 & 62.00 \\
\hline Patient group with and without $B R C A 1$ or $B R C A 2$ mutations & 51 & $44.80 \pm 10.97$ & 24.00 & 76.00 \\
\hline Control group & 55 & $44.00 \pm 8.11$ & 41.00 & 72.00 \\
\hline
\end{tabular}

SD: standard deviation. 
No statistically significant differences were detected between the genotype frequencies of the rs4485469, rs9646629, rs34739845, rs17069904, rs884205 and rs4941129 SNPs and metastasis in the patient group ( $p$ $>0.05$ ) (Table 4). Furthermore, there was no statistically significant difference found between metastasis status and the rs4485469, rs9646629, rs34739845, rs17069904, rs884205 and rs4941129 SNPs in the TNFRSF11A gene for patients both with and without $B R C A 1$ or $B R C A 2 \mathrm{mu}-$ tations (Table 5).

In patients with or without $B R C A 1$ or $B R C A 2$ mutations, no statistically significant differences were detected between estrogen receptor status, progesterone receptor status and CerbB2 status and the TNFRSF11A gene rs4485469, rs9646629, rs34739845, rs17069904, rs884205 and rs4941129 SNPs genotype frequencies (Table 6, Table 7 and Table 8).

Table 3. Comparison of genotype frequencies of TNFRSF11A single nucleotide polymorphisms between patient groups with and without $B R C A 1$ or $B R C A 2$ mutations.

\begin{tabular}{|c|c|c|c|c|}
\hline SNPs & Allele & $\mid \begin{array}{c}\text { Patients With } \\
\text { Mutations } \\
n(\%)\end{array}$ & $\begin{array}{c}\text { Patients } \\
\text { Without } \\
\text { Mutations } \\
n(\%) \\
\end{array}$ & $p$ Value \\
\hline \multirow{3}{*}{ rs4485459 } & AA & $10(43.5)$ & $4(14.3)$ & \multirow{3}{*}{0.059} \\
\hline & $\mathrm{AG}$ & $7(30.4)$ & $15(53.6)$ & \\
\hline & GG & $6(26.1)$ & $9(32.1)$ & \\
\hline \multirow{3}{*}{ rs9646629 } & GG & $11(47.8)$ & $8(28.6)$ & \multirow{3}{*}{0.367} \\
\hline & CG & $9(39.1)$ & $15(53.6)$ & \\
\hline & $\mathrm{CC}$ & $3(13.0)$ & $5(17.9)$ & \\
\hline \multirow{3}{*}{ rs34739845 } & AA & $18(78.3)$ & $22(78.6)$ & \multirow{3}{*}{0.632} \\
\hline & $\mathrm{AG}$ & $5(21.7)$ & $5(17.9)$ & \\
\hline & GG & $0(0.0)$ & $1(3.6)$ & \\
\hline \multirow{3}{*}{ rs17069904 } & GG & $17(73.9)$ & $23(82.1)$ & \multirow{3}{*}{0.491} \\
\hline & $\mathrm{AG}$ & $5(21.7)$ & $5(17.9)$ & \\
\hline & AA & $1(4.3)$ & $0(0.0)$ & \\
\hline \multirow{3}{*}{ rs 884205} & $\mathrm{CC}$ & $14(60.9)$ & $15(53.6)$ & \multirow{3}{*}{0.804} \\
\hline & $\mathrm{AC}$ & $7(30.4)$ & $11(39.3)$ & \\
\hline & AA & $2(8.7)$ & $2(7.1)$ & \\
\hline \multirow{3}{*}{ rs4941129 } & TT & $12(52.2)$ & $14(50.0)$ & \multirow{3}{*}{0.973} \\
\hline & CT & $10(43.5)$ & $13(46.4)$ & \\
\hline & $\mathrm{CC}$ & $1(4.3)$ & $1(3.6)$ & \\
\hline
\end{tabular}

SNPs: single nucleotide polymorphisms.
Table 2. Comparison of genotype frequencies of TNFRSF11A single nucleotide polymorphisms in patient and the control groups.

\begin{tabular}{|c|c|c|c|c|}
\hline SNPs & Allele & $\begin{array}{l}\text { Patient } \\
\text { Group } \\
n(\%)\end{array}$ & $\begin{array}{c}\text { Control } \\
\text { Group } \\
n(\%) \\
\end{array}$ & $p$ Value \\
\hline \multirow{3}{*}{ rs4941129 } & TT & $26(51.0)$ & $29(52.7)$ & \multirow{3}{*}{0.329} \\
\hline & $\mathrm{CT}$ & $23(45.1)$ & $20(36.4)$ & \\
\hline & $\mathrm{CC}$ & $2(3.9)$ & $6(10.9)$ & \\
\hline \multirow{3}{*}{ rs884205 } & $\mathrm{CC}$ & $29(56.9)$ & $35(63.6)$ & \multirow{3}{*}{0.104} \\
\hline & $\mathrm{AC}$ & $18(35.3)$ & $20(36.4)$ & \\
\hline & AA & $4(7.8)$ & $0(0.0)$ & \\
\hline \multirow{3}{*}{ rs17069904 } & GG & $40(78.4)$ & $49(89.1)$ & \multirow{3}{*}{0.297} \\
\hline & $\mathrm{AG}$ & $10(19.6)$ & $5(9.1)$ & \\
\hline & AA & $1(2.0)$ & $1(1.8)$ & \\
\hline \multirow{3}{*}{ rs34739845 } & AA & $40(78.4)$ & $36(65.5)$ & \multirow{3}{*}{0.331} \\
\hline & $\mathrm{AG}$ & $10(19.6)$ & $17(30.9)$ & \\
\hline & GG & $1(2.0)$ & $2(3.6)$ & \\
\hline \multirow{3}{*}{ rs9646629 } & GG & $19(37.3)$ & $26(47.3)$ & \multirow{3}{*}{0.546} \\
\hline & $\mathrm{CG}$ & $24(47.1)$ & $23(41.8)$ & \\
\hline & $\mathrm{CC}$ & $8(15.7)$ & $6(10.9)$ & \\
\hline \multirow{3}{*}{ rs4485469 } & AA & $14(27.5)$ & $13(23.6)$ & \multirow{3}{*}{0.820} \\
\hline & $\mathrm{AG}$ & $22(43.1)$ & $27(49.1)$ & \\
\hline & GG & $15(29.4)$ & $15(23.7)$ & \\
\hline
\end{tabular}

SNPs: single nucleotide polymorphisms.

a Patients with and without $B R C A 1$ and $B R C A 2$ mutations.

Table 4. Comparison of the relationship between the TNFRSF 11A single nucleotide polymorphisms and metastasis status in the patient group with and without $B R C A 1$ or $B R C A 2$ mutations.

\begin{tabular}{|c|c|c|c|c|}
\hline SNPs & Allele & $\begin{array}{c}\text { Patients } \\
\text { With Neg. } \\
\text { Metastasis } \\
n(\%)\end{array}$ & $\begin{array}{c}\text { Patients } \\
\text { With Pos. } \\
\text { Metastasis } \\
n(\%)\end{array}$ & $\begin{array}{c}p \\
\text { Value }\end{array}$ \\
\hline \multirow{3}{*}{ rs4941129 } & TT & $9(52.9)$ & $12(42.9)$ & \multirow{3}{*}{0.463} \\
\hline & $\mathrm{CT}$ & $7(41.2)$ & $15(53.6)$ & \\
\hline & $\mathrm{CC}$ & $1(5.9)$ & $1(3.6)$ & \\
\hline \multirow{3}{*}{ rs884205 } & $\mathrm{CC}$ & $7(41.2)$ & $19(67.9)$ & \multirow{3}{*}{0.352} \\
\hline & $\mathrm{AC}$ & $9(52.9)$ & $7(25.0)$ & \\
\hline & AA & $1(5.9)$ & $2(7.1)$ & \\
\hline \multirow{3}{*}{ rs17069904 } & GG & $13(76.5)$ & $23(82.1)$ & \multirow{3}{*}{0.718} \\
\hline & $\mathrm{AG}$ & $4(23.5)$ & $4(14.3)$ & \\
\hline & AA & $0(0.0)$ & $1(3.6)$ & \\
\hline \multirow{3}{*}{ rs34739845 } & AA & $12(70.6)$ & $24(85.7)$ & \multirow{3}{*}{0.471} \\
\hline & $\mathrm{AG}$ & $4(23.5)$ & $4(14.3)$ & \\
\hline & GG & $1(5.9)$ & $0(0.0)$ & \\
\hline \multirow{3}{*}{ rs9646629 } & GG & $3(17.6)$ & $13(46.4)$ & \multirow{3}{*}{0.348} \\
\hline & $\mathrm{CG}$ & $11(64.7)$ & $11(39.3)$ & \\
\hline & $\mathrm{CC}$ & $3(17.6)$ & $4(14.3)$ & \\
\hline \multirow{3}{*}{ rs4485469 } & AA & $4(23.5)$ & $10(35.7)$ & \multirow{3}{*}{0.365} \\
\hline & $\mathrm{AG}$ & $9(52.9)$ & $10(35.7)$ & \\
\hline & GG & $4(23.5)$ & $8(28.6)$ & \\
\hline
\end{tabular}

SNPs: single nucleotide polymorphisms Neg.: negative; Pos.; positive. 
BALKAN JOURNAL OF MEDICAL GENETICS

Özdemir K, Gürkan H, Demir S, Atli E, Özen Y, Sezer A, Tunçbilek N, Çicin I

Table 5. Comparison of the relationship between metastasis status and TNFRSF11A single nucleotide polymorphisms in the patient groups.

\begin{tabular}{|c|c|c|c|c|c|c|c|}
\hline \multirow[t]{2}{*}{ SNPs } & \multirow[t]{2}{*}{ Alleles } & \multicolumn{3}{|c|}{$\begin{array}{l}\text { Patients With } B R C A 1 \\
\text { or } B R C A 2 \text { Mutations }\end{array}$} & \multicolumn{3}{|c|}{$\begin{array}{l}\text { Patients Without } B R C A 1 \\
\text { or } B R C A 2 \text { Mutations }\end{array}$} \\
\hline & & $\begin{array}{c}\text { Negative } \\
\text { Metastasis } \\
n(\%)\end{array}$ & $\begin{array}{c}\text { Positive } \\
\text { Metastasis } \\
n(\%) \\
\end{array}$ & $p$ Value & $\begin{array}{c}\text { Negative } \\
\text { Metastasis } \\
n(\%)\end{array}$ & $\begin{array}{c}\text { Positive } \\
\text { Metastasis } \\
n(\%)\end{array}$ & $p$ Value \\
\hline \multirow{3}{*}{ rs4941129 } & $\mathrm{TT}$ & $3(50.0)$ & $4(36.4)$ & & $6(54.5)$ & $8(47.1)$ & \multirow{3}{*}{0.696} \\
\hline & $\mathrm{CT}$ & $2(33.3)$ & $7(63.6)$ & 0.156 & $5(45.5)$ & $8(47.1)$ & \\
\hline & $\mathrm{CC}$ & $1(16.7)$ & $0(0.0)$ & & $0(0.0)$ & $1(5.9)$ & \\
\hline \multirow{3}{*}{ rs884205 } & $\mathrm{CC}$ & $3(50.0)$ & $8(72.7)$ & & $4(36.4)$ & $11(64.7)$ & \multirow{3}{*}{0.338} \\
\hline & $\mathrm{AC}$ & $3(50.0)$ & $2(18.2)$ & 0.598 & $6(54.5)$ & $5(29.4)$ & \\
\hline & AA & $0(0.0)$ & $1(9.1)$ & & $1(9.1)$ & $1(5.9)$ & \\
\hline \multirow{3}{*}{ rs17069904 } & GG & $4(66.7)$ & $9(81.8)$ & & $9(81.8)$ & $14(82.4)$ & \multirow{3}{*}{0.971} \\
\hline & $\mathrm{AG}$ & $2(33.3)$ & $1(9.1)$ & 0.588 & $2(18.2)$ & $3(17.6)$ & \\
\hline & AA & $0(0.0)$ & $1(9.1)$ & & $0(0.0)$ & $0(0.0)$ & \\
\hline \multirow{3}{*}{ rs34739845 } & AA & $5(83.3)$ & $9(81.8)$ & & 7 (63.6) & $15(88.2)$ & \multirow{3}{*}{0.228} \\
\hline & $\mathrm{AG}$ & $1(16.7)$ & $2(18.2)$ & 0.724 & $3(27.3)$ & $2(11.8)$ & \\
\hline & GG & $0(0.0)$ & $0(0.0)$ & & $1(9.1)$ & $0(0.0)$ & \\
\hline \multirow{3}{*}{ rs9646629 } & GG & $2(33.3)$ & $6(54.5)$ & & $1(9.1)$ & 7 (41.2) & \multirow{3}{*}{0.161} \\
\hline & $\mathrm{CG}$ & $4(66.7)$ & $3(27.3)$ & 0.550 & 7 (63.6) & $8(47.1)$ & \\
\hline & $\mathrm{CC}$ & $0(0.0)$ & $2(18.2)$ & & $3(27.3)$ & $2(11.8)$ & \\
\hline \multirow{3}{*}{ rs4485469 } & AA & $4(66.7)$ & $6(54.5)$ & & $0(0.0)$ & $4(23.5)$ & \multirow{3}{*}{0.138} \\
\hline & $\mathrm{AG}$ & $1(16.7)$ & $3(27.3)$ & 0.158 & $8(72.7)$ & $7(41.2)$ & \\
\hline & GG & $1(16.7)$ & $2(18.2)$ & & $3(27.3)$ & $6(35.3)$ & \\
\hline
\end{tabular}

SNPs: single nucleotide polymorphisms.

Table 6. Comparison of the relationship between estrogen receptor status and TNFRSF11A single nucleotide polymorphisms in the patients groups.

\begin{tabular}{|c|c|c|c|c|c|c|c|}
\hline \multirow[t]{2}{*}{ SNPs } & \multirow[t]{2}{*}{ Alleles } & \multicolumn{3}{|c|}{$\begin{array}{l}\text { Patients With } B R C A 1 \text { or } \\
\text { BRCA2 Mutations }\end{array}$} & \multicolumn{3}{|c|}{$\begin{array}{l}\text { Patients Without } B R C A 1 \\
\text { or } B R C A 2 \text { Mutations }\end{array}$} \\
\hline & & $\begin{array}{l}\text { Negative } \\
\operatorname{ER} n(\%)\end{array}$ & $\begin{array}{c}\text { Positive } \\
\text { ER } n(\%)\end{array}$ & $p$ Value & $\begin{array}{l}\text { Negative } \\
\text { ER } n(\%)\end{array}$ & $\begin{array}{c}\text { Positive } \\
\text { ER } n(\%)\end{array}$ & $p$ Value \\
\hline \multirow{3}{*}{ rs4941129 } & TT & $2(25.0)$ & $4(50.0)$ & & $3(50.0)$ & $11(50.0)$ & \multirow{3}{*}{0.863} \\
\hline & $\mathrm{CT}$ & $6(75.0)$ & $3(37.5)$ & 0.100 & $3(50.0)$ & $10(45.5)$ & \\
\hline & $\mathrm{CC}$ & $0(0.0)$ & $1(12.5)$ & & $0(0.0)$ & $1(4.5)$ & \\
\hline \multirow{3}{*}{ rs 884205} & $\mathrm{CC}$ & $6(75.0)$ & $4(50.0)$ & & $3(50.0)$ & $12(54.5)$ & \multirow{3}{*}{0.675} \\
\hline & $\mathrm{AC}$ & $2(25.0)$ & $4(50.0)$ & 0.153 & $3(50.0)$ & $8(36.4)$ & \\
\hline & AA & $0(0.0)$ & $0(0.0)$ & & $0(0.0)$ & $2(9.1)$ & \\
\hline \multirow{3}{*}{ rs17069904 } & GG & $7(87.5)$ & $6(75.0)$ & & $5(83.3)$ & $18(81.8)$ & \multirow{3}{*}{0.932} \\
\hline & $\mathrm{AG}$ & $1(12.5)$ & $2(25.0)$ & 0.516 & $1(16.7)$ & $4(18.2)$ & \\
\hline & AA & $0(0.0)$ & $0(0.0)$ & & $0(0.0)$ & $0(0.0)$ & \\
\hline \multirow{3}{*}{ rs34739845 } & AA & $7(87.5)$ & $7(87.5)$ & & $5(83.3)$ & $17(77.3)$ & \multirow{3}{*}{0.860} \\
\hline & $\mathrm{AG}$ & $1(12.5)$ & $1(12.5)$ & 0.267 & $1(16.7)$ & $4(18.2)$ & \\
\hline & GG & $0(0.0)$ & $0(0.0)$ & & $0(0.0)$ & $1(4.5)$ & \\
\hline \multirow{3}{*}{ rs9646629 } & GG & $5(62.5)$ & $2(25.0)$ & & $1(16.7)$ & $7(31.8)$ & \multirow{3}{*}{0.223} \\
\hline & $\mathrm{CG}$ & $3(37.5)$ & $5(62.5)$ & 0.205 & $5(83.3)$ & $10(45.5)$ & \\
\hline & $\mathrm{CC}$ & $0(0.0)$ & $1(12.5)$ & & $0(0.0)$ & $5(22.7)$ & \\
\hline \multirow{3}{*}{ rs4485469 } & AA & $5(62.5)$ & $4(50.0)$ & & $0(0.0)$ & $4(18.2)$ & \multirow{3}{*}{0.512} \\
\hline & $\mathrm{AG}$ & $2(25.0)$ & $2(25.0)$ & 0.413 & $4(66.7)$ & $11(50.0)$ & \\
\hline & GG & $1(12.5)$ & $2(25.0)$ & & $2(33.3)$ & $7(31.8)$ & \\
\hline
\end{tabular}

SNPs: single nucleotide polymorphisms; ER: estrogen receptor. 
Table 7. Comparison of the relationship between progesterone receptor status and TNFRSF11A single nucleotide polymorphisms in the patient groups.

\begin{tabular}{|c|c|c|c|c|c|c|c|}
\hline \multirow[t]{2}{*}{ SNPs } & \multirow[t]{2}{*}{ Alleles } & \multicolumn{3}{|c|}{$\begin{array}{c}\text { Patients With } B R C A 1 \text { or } \\
\text { BRCA2 Mutations }\end{array}$} & \multicolumn{3}{|c|}{$\begin{array}{l}\text { Patients Without } B R C A 1 \\
\text { or } B R C A 2 \text { Mutations }\end{array}$} \\
\hline & & $\begin{array}{l}\text { Negative } \\
\operatorname{PR} n(\%)\end{array}$ & $\begin{array}{l}\text { Positive } \\
\text { PR } n(\%)\end{array}$ & $p$ Value & $\begin{array}{l}\text { Negative } \\
\operatorname{PR} n(\%)\end{array}$ & $\begin{array}{c}\text { Positive } \\
\text { PR } n(\%)\end{array}$ & $p$ Value \\
\hline \multirow{3}{*}{ rs 4941129} & TT & $4(36.4)$ & $2(40.0)$ & & $3(37.5)$ & $11(55.0)$ & \multirow{3}{*}{0.503} \\
\hline & $\mathrm{CT}$ & $7(63.6)$ & $2(40.0)$ & 0.085 & $5(62.5)$ & $8(40.0)$ & \\
\hline & $\mathrm{CC}$ & $0(0.0)$ & $1(20.0)$ & & $0(0.0)$ & $1(5.0)$ & \\
\hline \multirow{3}{*}{ rs884205 } & $\mathrm{CC}$ & $7(63.6)$ & $3(60.0)$ & & $4(50.0)$ & $11(55.0)$ & \multirow{3}{*}{0.562} \\
\hline & $\mathrm{AC}$ & $4(36.4)$ & $2(40.0)$ & 0.242 & $4(50.0)$ & $7(35.0)$ & \\
\hline & AA & $0(0.0)$ & $0(0.0)$ & & $0(0.0)$ & $2(10.0)$ & \\
\hline \multirow{3}{*}{ rs17069904 } & GG & $10(90.9)$ & $3(60.0)$ & & $5(62.5)$ & $18(90.0)$ & \multirow{3}{*}{0.086} \\
\hline & $\mathrm{AG}$ & $1(9.1)$ & $2(40.0)$ & 0.304 & $3(37.5)$ & $2(10.0)$ & \\
\hline & AA & $0(0.0)$ & $0(0.0)$ & & $0(0.0)$ & $0(0.0)$ & \\
\hline \multirow{3}{*}{ rs34739845 } & AA & $10(90.9)$ & $4(80.0)$ & & $6(75.0)$ & $16(80.0)$ & \multirow{3}{*}{0.691} \\
\hline & $\mathrm{AG}$ & $1(9.1)$ & $1(20.0)$ & 0.237 & $2(25.0)$ & $3(15.0)$ & \\
\hline & GG & $0(0.0)$ & $0(0.0)$ & & $0(0.0)$ & $1(5.0)$ & \\
\hline \multirow{3}{*}{ rs9646629 } & GG & $6(54.5)$ & $1(20.0)$ & & $3(37.5)$ & $5(25.0)$ & \multirow{3}{*}{0.290} \\
\hline & $\mathrm{CG}$ & $5(45.5)$ & $3(60.0)$ & 0.220 & $5(62.5)$ & $10(50.0)$ & \\
\hline & $\mathrm{CC}$ & $0(0.0)$ & $1(20.0)$ & & $0(0.0)$ & $5(25.0)$ & \\
\hline \multirow{3}{*}{ rs4485469 } & AA & $6(54.5)$ & $3(60.0)$ & & $0(0.0)$ & $4(20.0)$ & \multirow{3}{*}{0.069} \\
\hline & $\mathrm{AG}$ & $2(18.2)$ & $2(40.0)$ & 0.276 & $7(87.5)$ & $8(40.0)$ & \\
\hline & GG & $3(27.3)$ & $0(0.0)$ & & $1(12.5)$ & $8(40.0)$ & \\
\hline
\end{tabular}

SNPs: single nucleotide polymorphisms; PR: progesterone receptor.

Table 8. Comparison of the relationship between CerbB2 receptor status and TNFRSF11A single nucleotide polymorphisms in the patient groups.

\begin{tabular}{|c|c|c|c|c|c|c|c|}
\hline \multirow[t]{2}{*}{ SNPs } & \multirow[t]{2}{*}{ Alleles } & \multicolumn{3}{|c|}{$\begin{array}{l}\text { Patients With } B R C A 1 \\
\text { or } B R C A 2 \text { Mutations }\end{array}$} & \multicolumn{3}{|c|}{$\begin{array}{l}\text { Patients Without } B R C A 1 \\
\text { or } B R C A 2 \text { Mutations }\end{array}$} \\
\hline & & $\begin{array}{c}\text { Neg. CerB2 } \\
\text { Receptor } \\
n(\%)\end{array}$ & $\begin{array}{c}\text { Pos. CerB2 } \\
\text { Receptor } \\
n(\%)\end{array}$ & $p$ Value & $\begin{array}{c}\text { Neg. CerB2 } \\
\text { Receptor } \\
n(\%)\end{array}$ & $\begin{array}{c}\text { Pos. CerB2 } \\
\text { Receptor } \\
n(\%)\end{array}$ & $p$ Value \\
\hline \multirow{3}{*}{ rs4941129 } & TT & $4(50.0)$ & $2(28.6)$ & & $7(43.8)$ & $7(58.3)$ & \multirow{3}{*}{0.564} \\
\hline & $\mathrm{CT}$ & $3(37.5)$ & $5(71.4)$ & 0.250 & $8(50.0)$ & $5(41.7)$ & \\
\hline & $\mathrm{CC}$ & $1(12.5)$ & $0(0.0)$ & & $1(6.3)$ & $0(0.0)$ & \\
\hline \multirow{3}{*}{ rs884205 } & $\mathrm{CC}$ & $4(50.0)$ & $5(71.4)$ & & $8(50.0)$ & $7(58.3)$ & \multirow{3}{*}{0.852} \\
\hline & $\mathrm{AC}$ & $4(50.0)$ & $2(28.6)$ & 0.207 & $7(43.8)$ & $4(33.3)$ & \\
\hline & AA & $0(0.0)$ & $0(0.0)$ & & $1(6.3)$ & $1(8.3)$ & \\
\hline \multirow{3}{*}{ rs17069904 } & GG & $6(75.0)$ & $6(85.7)$ & & $14(87.5)$ & $9(75.0)$ & \multirow{3}{*}{0.393} \\
\hline & $\mathrm{AG}$ & $2(25.0)$ & $1(14.3)$ & 0.661 & $2(12.5)$ & $3(25.0)$ & \\
\hline & AA & $0(0.0)$ & $0(0.0)$ & & $0(0.0)$ & $0(0.0)$ & \\
\hline \multirow{3}{*}{ rs34739845 } & AA & $7(87.5)$ & $6(85.7)$ & & $12(75.0)$ & $10(83.3)$ & \multirow{3}{*}{0.292} \\
\hline & $\mathrm{AG}$ & $1(12.5)$ & $1(14.3)$ & 0.407 & $4(25.0)$ & $1(8.3)$ & \\
\hline & GG & $0(0.0)$ & $0(0.0)$ & & $0(0.0)$ & $1(8.3)$ & \\
\hline \multirow{3}{*}{ rs9646629 } & GG & $3(37.5)$ & $3(42.9)$ & & $4(25.0)$ & $4(33.3)$ & \multirow{3}{*}{0.890} \\
\hline & $\mathrm{CG}$ & $5(62.5)$ & $3(42.9)$ & 0.278 & $9(56.3)$ & $6(50.0)$ & \\
\hline & $\mathrm{CC}$ & $0(0.0)$ & $1(14.3)$ & & $3(18.8)$ & $2(16.7)$ & \\
\hline \multirow{3}{*}{ rs 4485469} & AA & $4(50.0)$ & $4(57.1)$ & & $2(12.5)$ & $2(16.7)$ & \multirow{3}{*}{0.540} \\
\hline & $\mathrm{AG}$ & $1(12.5)$ & $3(42.9)$ & 0.291 & $10(62.5)$ & $5(41.7)$ & \\
\hline & GG & $3(37.5)$ & $0(0.0)$ & & $4(25.0)$ & $5(41.7)$ & \\
\hline
\end{tabular}

SNPs: single nucleotide polymorphisms; Neg.: negative; Pos.: positive. 


\section{DISCUSSION}

It is now thought that the results of genetic studies will provide insight into new approaches for the treatment of breast cancer, which is a very important health problem with significant mortality rates, especially among women. For this purpose, extensive studies with genes, proteins and enzymes have been performed to examine the molecular mechanisms that play a role in the pathogenesis of breast cancer $[1,19]$. However, it is thought that there are still many mechanisms that are yet to be discovered. In recent years, the rising number of reports about the newly discovered genes and proteins have not only been focused on the molecular mechanisms of cancer, but also on the basis for developing approaches to both detect and treatment of cancer [20]. TNFRSF11A and RANKL are genes that are functionally linked, and they are often considered together in terms of their role in determining various traits. $R A N K L$ and receptor activator of nuclear $\kappa \mathrm{B}(R A N K)$ genes are essential for osteoclast activation and development [13]. In addition, it has been reported that the $R A N K$ gene contributes to breast tumorigenesis because it is expressed in mammary gland cells and controls lactation development during pregnancy $[21,22]$.

Although many genes that influence breast cancer have been investigated to date, there have been a limited number of studies on the effect of the TNFRSF11A gene on breast cancer. The results of genome-wide association studies conducted by the Cancer and Genetics Markers of Susceptibility (CGEMS) initiative have suggested that the common genetic variation (rs7226991) near the 5' terminal of TNFRSF11A was associated with breast cancer risk $[8,23]$. Several common breast cancer susceptibility alleles, identified by genome-wide association studies and in different populations, have also been associated with breast cancer risk among $B R C A 1$ and/or $B R C A 2$ mutation carriers [24].

Regarding these developments, rs7226991 genotypes have been detected in two Spanish case-control studies. Bonifaci et al. [8] revealed that rs7226991 was associated with a risk of breast cancer among the general Spanish population ( $B R C A 1$ or $B R C A 2$ ) along with $B R C A 2$ mutation carriers from different populations, which is consistent with the above observations. In addition, the results of the study exhibited effects parallel to those shown in the CGEMS results $[8,25,26]$.

In their study, Sigl et al. [17]. indicated that a total of 19 SNPs genotyped in or near the TNFRSF11A gene. In this particular study, it was reported that TNFRSF $11 A$ possibly contributes to both menarche and the natural onset of menopause, and early menarche and natural menopause age have been shown to increase the risk of breast cancer.
Similar to our results, no statistically significant difference was found for rs17069904, rs9646629 and rs884205 SNPs. Sigl et al. [17] also identified the role of the loci responsible for encoding TNFRSF11A as a genetic regulator of breast cancer risk in women with hereditary $B R C A 1$ mutations, as $R A N K / R A N K L$ significantly affected breast car-cinogenesis in mice with $B R C A 1$ mutation carriers. Six SNPs (rs9646629, rs4485469, rs34739845, rs4941129, rs17069904 and rs884205) have been reported to be significantly associated with the risk of breast cancer in ERnegative or triple-negative $B R C A 1$ mutation carriers; such a determination was made based on the consideration of 15,200 BRCA1 and 8200 BRCA2 mutation carriers using Collaborative Oncological Gene-environment Study (iCOGS) data in which 51 TNFRSF11A SNPs were genotyped. In addition, Sigl et al. [17] suggested that two SNPs in the TNFRSF11A gene (rs884205 and rs4485469) were significantly associated with breast cancer risk $(p<0.05)$ in BRCA1 and BRCA2 mutation carriers.

In our study, we found that TNFRSF11A rs4485469 SNP has a borderline significance between the patient group with and without $B R C A 1$ or $B R C A 2$ mutation ( $p=$ 0.059 ), compatible with the results of the study performed by Sigl et al. [17]. We anticipate that statistically significant results can be obtained if the study is repeated with an increased number of patients.

A limited number of studies in the literature have reported on the TNFRSF11A gene and its effect on the risk of developing breast cancer. Rao et al. [27] aimed to determine the effect of $R A N K$ signaling in breast cancer formation based on previous findings, showing that RANK plays an important role in mammary gland development and progestin-induced breast carcinogenesis. In their studies, in which they performed a multi-faceted analysis of the $R A N K / R A N K L$ system, Rao et al. [27] reported that the SNPs in the TNFRSF11A gene that increased RANK expression levels, were associated with an increased risk of breast cancer development in women with $B R C A 1$ or $B R C A 2$ mutations. They also suggested that developing breast cancer against the background of $B R C A 1$ or $B R C A 2$ mutations can be prevented by blocking $R A N K / R A N K L$ signaling. These findings may pave the way for a new era of breast cancer prophylaxis by changing the lives of millions of women worldwide [27]. With respect to our own findings, we predict that the investigation of the effects of the TNFRSF11A gene variations on breast cancer development risk in $B R C A 1$ and/or $B R C A 2$ mutation carrier breast cancer patients in different populations will contribute to refining the proper treatment protocols. 


\section{CONCLUSIONS}

In our study, we investigated the effects of rs4485469, rs9646629, rs34739845, rs17069904, rs884205 and rs4941129 SNPs in the TNFRSF11A gene on the risk of developing breast cancer among patients with BRCA1 and/or BRCA2 variations living in the Trakya region of Turkey. Among the TNFRSF11A polymorphisms that we included in our study, the rs4485469 SNP was found to be significantly borderline between the patient group with and without $B R C A 1$ or $B R C A 2$ mutations.

Differences in genetic study results related to the role of TNFRSF 11A SNPs in the development process of breast cancer may be due to ethnic differences between populations, the inclusion criteria of the patients, and the different sample numbers. Thus, we suggest that the relationship between TNFRSF11A polymorphisms and the risk of breast cancer development by increasing the sample size and studying in different populations with a similar methodology will make a significant contribution to the literature.

Acknowledgments. This study was externally peerreviewed. Author's contributions: H. Gürkan conceived the study; H. Gürkan and K. Özdemir designed the study; K. Özdemir and Y. Özen were responsible for resources; A. Sezer and İ. Çicin were responsible for the materials; H. Gürkan, K. Özdemir, S. Demir and N. Tunçbilek collected the data and/or processing; H. Gürkan, K. Özdemir, S. Demir and E. Atli analyzed and interpreted the data; H. Gürkan, K. Özdemir and S. Demir reviewed the literature; H. Gürkan, S. Demir, A. Sezer, İ. Çicin and N. Tunçbilek wrote the manuscript.

Declaration of Interest. The authors report no conflicts of interest. The authors alone are responsible for the content and writing of this article.

Funding. This study was funded by the Trakya University Scientific Research Projects Unit [TÜBAP-2016-252].

\section{REFERENCES}

1. Yararbas K, Atalay PB. Association of E-selectin S128R polymorphism with hereditary breast carcinoma susceptibility in Turkish patients without BRCA1/2 germline mutations. Balkan J Med Genet. 2018; 21(1): 27-32.

2. Armstrong N, Ryder S, Forbes C, Ross J, Quek RGw. A systematic review of the international prevalence of $B R C A$ mutation in breast cancer. Clin Epidemiol. 2019;11:543-561.
3. Hughes DC. Use of association studies to define genetic modifiers of breast cancer risk in $B R C A 1$ and BRCA2 mutation carriers. Fam Cancer. 2008; 7(3): 233-244.

4. Wittersheim M, Büttner R, Markiefka B. Genotype/ phenotype correlations in patients with hereditary breast cancer. Breast Care (Basel). 2015; 10(1): 2226.

5. Hughes DJ, Ginolhac SM, Coupier I, Barjhoux L, Gaborieau V, Bressac-de-Paillerets B, et al. Breast cancer risk in BRCA1 and BRCA2 mutation carriers and poly-glutamine repeat length in the AIB1 gene. Int J Cancer. 2005; 117(2): 230-233.

6. Pristauz G, Petru E, Stacher E, Geigl JB, Schwarzbraun T, Tsybrovskyy O, et al. Androgen receptor expression in breast cancer patients tested for BRCA1 and BRCA2 mutations. Histopathology. 2010; 57(6): 877-884.

7. Ricks-Santi LJ, Sucheston LE, Yang Y, Freudenheim JL, Isaacs CJ, Schwartz MD, et al. Association of Rad51 polymorphism with DNA repair in BRCA1 mutation carriers and sporadic breast cancer risk. BMC Cancer. 2011; 11: 278.

8. Bonifaci N, Palafox M, Pellegrini P, Osorio A, Benítez J, Peterlongo P, et al. Evidence for a link between TNFRSF11A and risk of breast cancer. Breast Cancer Res Treat. 2011; 129(3): 947-954.

9. Kim K, Kim JH, Lee J, Jin HM, Lee SH, Fisher DE, et al. Nuclear factor of activated T cells $\mathrm{c} 1$ induces osteoclast-associated receptor gene expression during tumor necrosis factor-related activation-induced cytokine-mediated osteoclastogenesis. J Biol Chem. 2005; 280(42): 35209-35216.

10. Geerts D, Chopra C, Connelly L. Osteoprotegerin: Relationship to breast cancer risk and prognosis. Front Oncol. 2020; 10: 462.

11. Boyce BF, Xing L. Biology of RANK, RANKL, and osteoprotegerin. Arthritis Res Ther. 2007; 9(Suppl 1): S1.

12. Willard D, Chen WJ, Barrett G, Blackburn K, Bynum J, Consler T, et al. Expression, purification, and characterization of the human receptor activator of NF-kappaB ligand (RANKL) extracellular domain. Protein Expr Purif. 2000; 20(1): 48-57.

13. Walsh MC, Choi Y. Biology of the RANKL-RANKOPG system in immunity, bone, and beyond. Front Immunol. 2014; 5: 511.

14. Sigl V, Jones LP, Penninger JM. RANKL/RANK: From bone loss to the prevention of breast cancer. Open Biol. 2016; 6(11): 160230. 
15. Kiesel L, Kohl A. Role of the RANK/RANKL pathway in breast cancer. Maturitas. 2016; 86: 10-16.

16. Renema N, Navet B, Heymann MF, Lezot F, Heymann D. RANK-RANKL signalling in cancer. Biosci Rep. 2016; 36(4): e00366.

17. Sigl V, Owusu Boaitey K, Joshi PA, Kavirayani A, Wirnsberger G, Novatchkova M, et al. RANKL/ RANK control BRCA1 mutation-driven mammary tumors. Nature. 2016; 26(7): 761-774.

18. Azim HA Jr, Peccatori FA, Brohée S, Branstetter D, Loi S, Viale G, et al. RANK ligand (RANKL) expression in young breast cancer patients and during pregnancy. Breast Cancer Res. 2015; 17: 24.

19. van Dam PA, Verhoeven Y, Trinh XB, Wouters A, Lardon F, Prenen H, et al. RANK/RANKL signaling inhibition may improve the effectiveness of checkpoint blockade in cancer treatment. Crit Rev Oncol Hematol. 2019; 133: 85-91.

20. Fardmanesh H, Shekari M, Movafagh A, Alizadeh Shargh S, Poursadegh Zonouzi AA, Shakerizadeh S. Up-regulation of the double-stranded RNA binding protein DGCR8 in invasive ductal breast carcinoma. Gene. 2016; 581(2): 146-151.

21. Toriola AT, Appleton CM, Zong X, Luo J, Weilbaecher K, Tamimi RM, et al. Circulating receptor activator of nuclear factor- $\kappa \mathrm{B}$ (RANK), RANK ligand (RANKL), and mammographic density in premenopausal women. Cancer Prev Res (Phila). 2018; 11(12): 789-796.
22. Lu Y, Liu P, Recker RR, Deng HW, Dvornyk V. TNFRSF11A and TNFSF11 are associated with age at menarche and natural menopause in white women. Menopause. 2010; 17(5): 1048-1054.

23. Chung PY, Beyens G, Riches PL, Van Wesenbeeck $\mathrm{L}$, de Freitas F, Jennes $\mathrm{K}$, et al. Genetic variation in the TNFRSF11A gene encoding RANK is associated with susceptibility to Paget's disease of bone. Bone Miner Res. 2010; 25(12): 2592-2605.

24. Lilyquist J, Ruddy KJ, Vachon CM, Couch FJ. Common genetic variation and breast cancer risk-past, present, and future. Cancer Epidemiol Biomarkers Prev. 2018; 27(4): 380-394.

25. Gayán J, Galan JJ, González-Pérez A, Sáez ME, Martínez-Larrad MT, Zabena C, et al. Genetic structure of the Spanish population. BMC Genomics. 2010; 11: 326 .

26. Ney JT, Juhasz-Boess I, Gruenhage F, Graeber S, Bohle RM, Pfreundschuh M, et al. Genetic polymorphism of the OPG gene associated with breast cancer. BMC Cancer. 2013; 13: 40.

27. Rao S, Cronin SJF, Sigl V, Penninger JM. RANKL and RANK: From mammalian physiology to cancer treatment. Trends Cell Biol. 2018; 28(3): 213-223. 